Clinical Study:

\title{
Hemorrhages Seen on Brain Images After Mild Traumatic Brain Injury May Increase the Risk of Post-concussion Syndrome
}

\author{
Seddigheh Eslamparast ${ }^{1}$ (D) , Zoheir Reihanian ${ }^{2,3}$ (D), Sara Ramezani ${ }^{2,1^{*}}$ (D) \\ 1. Guilan Road Trauma Research Center, Guilan University of Medical Sciences, Rasht, Iran \\ 2. Neuroscience Research Center, School of Medicine, Guilan University of Medical Sciences, Rasht, Iran \\ 3. Department of Neurosurgery, School of Medicine, Guilan University of Medical Sciences, Rasht, Iran
}

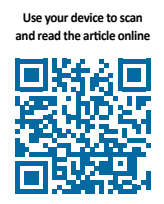

Cftation: Eslamparast S, Reihanian Z, Ramezani S. Hemorrhages Seen on Brain Images After Mild Traumatic Brain Injury May Increase the Risk of Post-concussion Syndrome. Iran J Neurosurg. 2020; 6(2):73-82. http://dx.doi.org/10.32598/irjns.6.2.4

doi: : http://dx.doi.org/10.32598/irjns.6.2.4

\section{(c) (i) (s)}

\section{Article info:}

Received: 08 Dec 2019

Accepted: 03 Feb 2020

Available Online: 01 Apr 2020
Keywords:

Mild traumatic brain injury,

Post-Concussion Syndrome,

Neuroimaging, Risk factors

\begin{abstract}
A B STRACT
Background and Aim: Mild Traumatic Brain Injury (mTBI) mostly develops the symptoms that may persist for over three months known as Post-Concussion Syndrome (PCS). However, the PCS potential risk for mTBI victims is not well-identified. Here, we investigated the putative risk factors of PCS.

Methods and Materials/Patients: In a cross-sectional study, we collected (HIS) the demographic, clinical, and radiological data using the hospital information system in 388 mTBI patients who passed at least 3 months since the onset of their injury and referred to Poursina hospital from March 2017 to December 2018. The patients were examined to diagnose PCS by a general physician using the phone interview via the Rivermead Post-concussion Symptoms Questionnaire (RPQ). The subjects were separated into groups with and without PCS. Data were analyzed by parametric t-test, Chi-square test and multiple logistic regression.

Results: One-hundred ninety one out of $388 \mathrm{mTBI}$ patients consented to complete the RPQ and around $59 \%$ of cases experienced PCS. There was no significant difference in the demographic variables and past medical history between groups. However, the previous psychological disease was particularly associated with PCS $(P>0.043)$. Length of hospitalization, functional outcome during discharge, and post-resuscitation consciousness did not show any significant association with PCS $(\mathrm{P}<0.05)$. Interestingly, initial abnormal brain scan, fronto-temporal lesion, and accompanied hematoma (hemorrhages) were identified as risk factors of mTBI-induced PCS. The risk of PCS was found to increase by 7.2 times in mTBI patients demonstrated as an abnormality in their initial brain scans $(P<0.001)$. A directly proportional relationship was found between the occurrence of the syndrome and the fronto-temporal lesion $(P<0.017)$. Accompanied hematoma enhanced the risk of PCS by 2.6 times $(\mathrm{P}<0.04)$.
\end{abstract}

Conclusion: This study emphasized the significance of early brain scan data for the prediction of PCS and the necessity of proper follow-up care for the at-risk population. The reported data from this study might be applied as an objective trajectory to measure PCS in those who simulated PCS for the litigation

\section{* Corresponding Author:}

Sara Ramezani, PhD.

Address: Guilan Road Trauma Research Center, Guilan University of Medical Sciences, Rasht, Iran

Tel: +98 (133) 3322444

E-mail:s.ramezanislp@gmail.com 


\section{Highlights}

- Over half of mild traumatic brain injury patients had PCS.

- Pathology on brain scans at first 48 hours of admission predicted PCS.

- The fronto-temporal lesion was the second predictor of PCS.

- Accompanied hematoma had a predictive potential for PCS.

- The history of psychological disorder was more in the PCS group than the non-PCS group.

- Patients with and without PCS were not different in demographic characteristics.

\section{Plain Language Summary}

Mild Traumatic Brain Injury (mTBI) is more prevalent than other severities of brain injuries and is characterized by the loss of consciousness for less than 30 min with a Glasgow Coma Scale (GCS) score from 13 to 15. Although the initial brain scans may be normal in the patients with $\mathrm{mTBI}$, the identifiable pathology might be detected in their neuroimaging findings. Some patients experience persistent symptoms called Post-concussion Syndrome (PCS) that may disappear soon after injury. Here, we aimed to investigate the potential risk of PCS in mTBI patients. For this purpose, we collected the personal record of demographic, clinical, and radiological data of $388 \mathrm{mTBI}$ patients who had been referred to Poursina hospital from March 2017 to December 2018 using the Hospital Information System (HIS). Follow-up calls were appointed and done for patients with the diagnosis of PCS at least three months after the injury. Rivermead Post-concussion Symptoms Questionnaire (RPQ) was filled in for the patients during the follow-up call. The patients were then divided into 2 groups according to the presence or absence of PCS. After statistical analysis, it has been found that none of the patients' demographic and clinical variables, including age, gender, and functional outcome during the discharge, accompanied traumatic injuries, and past medical history was associated with PCS. However, there was a pronounced association between previous psychological disease and the risk of PCS. Another finding from this study was that the initial abnormal brain scan was a profound risk factor for PCS development. Likewise, the fronto-temporal lesion and accompanied hematoma were identified as second and third predictors of PCS, respectively. It seems that emergency professionals need to seriously consider early brain scans for the prognosis of PCS and follow-up care of the population at risk. Therefore, neuroimaging can be used as an early objective measure to help differentiating true PCS from malinjuries and may have medico-legal values.

\section{Introduction}

raumatic Brain Injury (TBI) causes a collection of socio-psycho-economic problems in human societies. Trauma-induced brain damage is the leading cause of death and disability in people younger than 45 accounting for the most active part of society [1]. It has been shown that TBI leads to persisting neurobehavioral disorders and minor TBI especially brings about cognitive and emotional sequels [2]. Mild TBI (mTBI) accounts for $90 \%$ of traumatic brain injuries. An increasing prevalence of mTBI in the world has been revealed to be accompanied by significant socioeconomic sequels and considerable morbidity [3-5]. Despite the absence of any abnormalities on traditional neuroradiologic examinations in the early phase of mTBI, a substantial number of patients complain about post-traumatic neuropsychological dysfunction a few weeks later [6].

In the first weeks following $\mathrm{mTBI}$, many patients suffer from post-concussion symptoms including physical symptoms (i.e. headaches, dizziness, blurred vision, fatigue, and sleep disturbances), cognitive deficits (i.e. poor memory, attention, and executive difficulties), and behavioral/emotional symptoms (i.e. depression, irritability, anxiety disorders, emotional disability) [7]. These symptoms will spontaneously diminish for most patients [8], but for a subset of patients estimated between $5 \%$ $43 \%$, the symptoms last for over months and sometimes even longer [9-14]. When a set of symptoms persists for over three months, it is often referred to as Post- 
concussion Syndrome (PCS). The most applicable criteria for the diagnosis of PCS are those that were specified in the International Classification of Diseases (ICD-10) [15] and the Diagnostic and Statistical Manual of Mental Disorders (DSM-IV) [10]. A frequently used instrument to assess the presence and severity of PCS is the Rivermead Post-concussion Symptoms Questionnaire (RPQ) [16].

Sustained neuropsychiatric and neuropsychological sequel such as persistent headaches, nausea, attention, and memory impairments as well as personality changes and sleep disorders for more than 3 months called PCS may be clinically identified. However, intracranial abnormalities in neuroimaging studies rarely reveal consistent brain changes to explain these problems [17]. Nevertheless, evidence has indicated that subtle white matter disruption detected by diffuse tensor imaging is associated with PCS in minor TBI [18]. Indeed, the signs associated with $\mathrm{mTBI}$ are difficult to quantify at hospitalization using routine lab neuropsychological tests during the acute phase of injury. Nevertheless, the quality of life in mTBI patients may be significantly impaired following the discharge, suggesting that there are some problems to be detected and resolved.

Generally, emergency physicians treating the patients often make therapeutic decisions based on the prognosis obtained from clinical assessment during acute phase [19]. It is obvious that identifying patients at the risk of PCS development as well as timely decisions to manage mTBI-induced subsequences and follow-up care of patients at the risk of PCS are very critical for optimizing outcomes of $\mathrm{mTBI}$. Therefore, knowing the demographic, clinical, and paraclinical parameters of TBI patients is very essential for predicting TBI outcome and detecting the population at risk of PCS. Hence, this study aimed to investigate the risk factors of PCS in patients with mild TBI who referred to Poursina hospital in Rasht, in the north of Iran.

\section{Methods and Materials/Patients}

A cross-sectional study was designed. All patients with $\mathrm{mTBI}$ who had been referred to Poursina Hospital in Rasht from March 2017 to December 2018 with at least 3 months of their TBI onset entered in this study. All of the demographic, clinical, and radiological data for all TBI victims was collected through the Hospital Information System (HIS). Clinical and TBI-related data included post-resuscitation Glasgow Coma Scale (GCS) score, cause of TBI, length of hospitalization, accompanied traumatic injuries, the functional outcome at discharge based on Glasgow Outcome Scale (GOS) score, past medical history such as cancer, cardiovascular, endocrine, orthopedic, neurological, blood, gastrointestinal and kidney diseases. The previous mental illness was specifically investigated as a distinct variable. Radiological data consists of the information based on the presence or absence of abnormality in brain CT and structural MRI scans at maximum the first 48 hours after head trauma. Specifically, plurality, type, and locus of the brain lesions were interpreted using MRI scans by a neuroradiologist unaware of the neurological outcomes after brain trauma.

The findings were entered on a code sheet that specified the plurality of the brain lesion; anatomic location including involved cerebral lobe(s) in the focal and multifocal lesions as well as injury type including a skull fracture and intracranial lesions such as pneumocephalus, contusion, Intracerebral Hemorrhage (ICH), subarachnoid hemorrhage (SAH), subdural hematoma (SDH), Epidural Hematoma (EDH) of each focal abnormal intensity and accompanied hematoma defined as a kind of hematoma plus other hemorrhagic lesions or contusion or skull fracture or pneumocephalus or combination of some or all them. All patients who had passed 3 months or more with mild TBI were examined to detect PCS. The development of PCS was determined via an interview with the patients by a general physician using the phone to complete RPQ according to the patient's statements. A score of 12 or more on the RPQ questionnaire was considered as PCS [20]. Data analysis was done by SPSS software version 22. The subjects were assigned to 2 groups with and without PCS. A parametric t-test was carried out for the comparison of groups in terms of quantitative variables. A Chi-square test was utilized to compare the groups regarding the qualitative variables. Multiple logistic regression was applied to determine the PCS predictors.

\section{Results}

The survey demonstrated that 388 people who had been referred to Poursina hospital between March 2017 and December 2018 were mTBI. However, only 191 patients gave consent to complete the RPQ for the detection of PCS. It was found that 183 patients did not agree to respond to the questions of RPQ, and 14 persons had died before the investigator contacted the patient for the determination of PCS using RPQ (Figure 1). Thus, descriptive analysis was carried out for the total population of this study including $388 \mathrm{mTBI}$ patients. The average age of $\mathrm{mTBI}$ victims was $43.28 \pm 22.15$ years. Also, the average length of hospitalization was $4.55 \pm 3.52$ days. About $39.9 \%$ of patients had a GCS score of 15 . Based 
Table 1. Description and comparison of characteristics of mTBI patients according to PCS

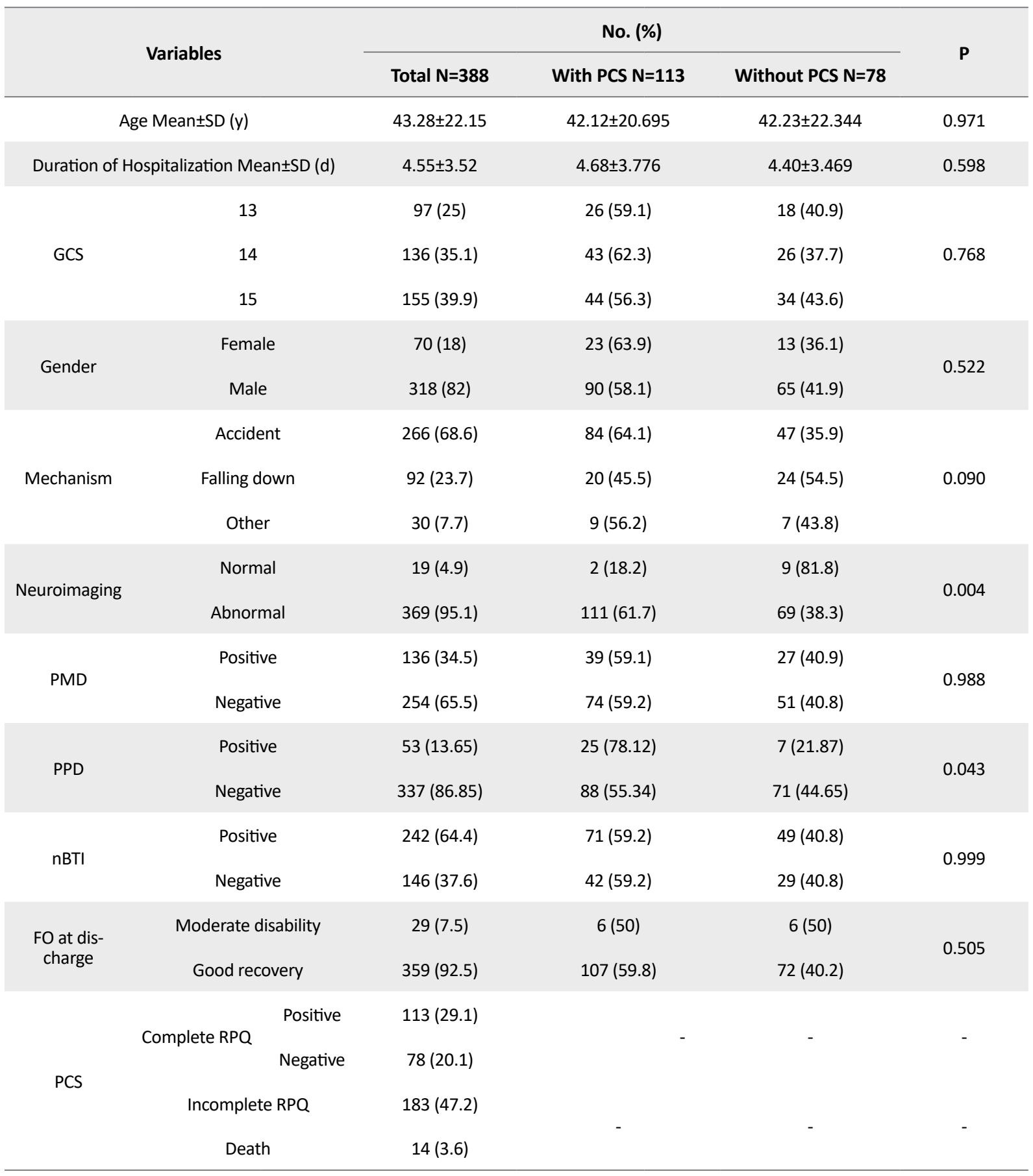

GCS: Glasgow coma scale; PMD: Past medical disease, PPD: Previous psychological/mental disorders, nBTI: non-brain traumatic injury; FO: Functional outcome; PCS: Post-concussion syndrome, SD: Standard deviation.

on the results, GCS score 14 and 13 were observed in $35.1 \%$ and $25 \%$ of subjects, respectively. Approximately, $82 \%$ of $\mathrm{mTBI}$ patients were male and $18 \%$ of them were female. Our findings exhibited that road traffic accident was a more common mechanism of TBI (68.6\%) in the current investigation. According to the brain CT scan data, $95.1 \%$ of the brain CT scans had pathological lesions and the rest was normal. About $34.5 \%$ of patients had a variety of past medical diseases. On basis of the GOS findings, $92.5 \%$ of patients recovered and $7.5 \%$ of them were discharged with moderate disability. 
Table 2. Comparison of frequency of lesion side, sites and types between patients with abnormal brain imaging with and without PCS

\begin{tabular}{|c|c|c|c|c|}
\hline \multirow[b]{2}{*}{ Variables } & \multicolumn{3}{|c|}{ No. (\%) } & \multirow[b]{2}{*}{$\mathbf{P}$} \\
\hline & $\begin{array}{l}\text { All Patients With Abnor- } \\
\text { mal Brain Scan N=369 }\end{array}$ & $\begin{array}{c}\text { PCS Group With } \\
\text { Abnormal Brain Scan } \\
\text { N=111 }\end{array}$ & $\begin{array}{l}\text { Non-PCS Group With Ab- } \\
\text { normal Brain Scan N=69 }\end{array}$ & \\
\hline Brain lesion type & & & & 0.025 \\
\hline Pneumocephalus & $4(1.08)$ & - & - & \\
\hline Skull fracture +Contusion & $8(2.16)$ & - & $1(1.44)$ & \\
\hline contusion & $74(20.05)$ & - & $3(4.34)$ & \\
\hline $\mathrm{ICH}$ & $8(2.16)$ & $7(6.30)$ & $4(5.79)$ & \\
\hline SAH & $33(8.94)$ & $9(8.1)$ & $7(10.14)$ & \\
\hline SDH & $60(16.26)$ & $15(13.51)$ & $14(20.28)$ & \\
\hline $\mathrm{EDH}$ & $84(22.76)$ & $22(19.81)$ & $21(30.43)$ & \\
\hline Accompanied hematoma & $98(26.55)$ & $58(52.25)$ & $19(27.53)$ & \\
\hline Brain lesion site & & & & 0.001 \\
\hline Frontal & $136(36.85)$ & $41(36.93)$ & $22(31.88)$ & \\
\hline Temporal & $89(24.11)$ & 11 (9.9) & $16(23.18)$ & \\
\hline Parietal & $44(11.92)$ & $1(0.9)$ & $12(17.39)$ & \\
\hline Tempo-parietal & $18(4.87)$ & $2(1.8)$ & $11(15.94)$ & \\
\hline Fronto-parietal & $21(5.69)$ & $5(4.5)$ & $1(1.44)$ & \\
\hline Fronto-temporal & $44(11.92)$ & $38(34.23)$ & $4(5.79)$ & \\
\hline Fronto-tempo-parietal & $17(4.6)$ & $13(11.71)$ & $3(4.34)$ & \\
\hline Lesion plurality & & & & 0.032 \\
\hline Focal & $271(73.44)$ & $58(52.25)$ & $50(72.46)$ & \\
\hline Multifocal & $98(26.55)$ & $53(47.74)$ & $19(27.53)$ & \\
\hline
\end{tabular}

Of 191 patients with $\mathrm{mTB}$ I who had completed the RPQ, $59.17 \%$ of people had PCS (Table 1 ).

As shown in Table 1, there was no significant difference in the mean age $(P>0.971)$ and the length of hospitalization $(P<0.958)$ between $\mathrm{mTB}$ patients with and without PCS. An insignificant difference in the frequency of gender was seen between the groups with and without PCS $(P<0.522)$. Among patients with an abnormal brain scan, about $61.7 \%$ of them had PCS. Nearly, $18.2 \%$ of those with normal brain scan experienced PCS. The percentage of abnormal findings on the brain scans of the PCS group was substantially higher than the non-PCS group
$(P<0.004)$. There was no significant relationship between the appearance of PCS and past medical disease $(\mathrm{P}<0.988)$. About $61.7 \%$ of patients with an abnormal brain scan had PCS. Nearby $18.2 \%$ of those with normal brain scans experienced PCS. The percentage of abnormal findings on the brain scans of the PCS group was substantially higher than the non-PCS group $(P<0.004)$. There was no significant relationship between the appearance of PCS and past medical disease $(P<0.988)$. No significant difference was observed between the 2 groups with and without PCS in terms of post-resuscitation GCS scores $(P<0.768)$ and functional outcome at discharge on GOS $(P<0.505)$ (Table 1$)$. 
Table 3. The results of Multiple Logistic Regression to predict PCS appearance after mTBI

\begin{tabular}{llcccccc}
\hline & variables & B & SE & df & P & OR & Cl \\
\hline \multirow{3}{*}{ Step 2 } & Accompanied hematoma & 1.035 & 1.59 & 1 & 0.04 & 2.68 & $0.82-5.61$ \\
& Fronto-temporal lesion & 1.224 & 1.165 & 1 & 0.017 & 4.15 & $1.1-7.06$ \\
& Abnormal brain scan & 1.890 & 0.708 & 1 & 0.001 & 7.239 & $1.43-11.59$ \\
\hline
\end{tabular}

SE: Standard Error; df: Degree freedom; OR: Odd ratio; Cl: Confidence interval; PCS: Post-concussion Syndrome.

The results in Table 2 clarified the frontal lesions $(36.85 \%)$ as a more common brain lesion site in all patients with an abnormal brain scan. In this population, accompanied hematoma (26.55\%), EDH (22.76\%), contusions (20.05\%) were the most prevalent lesion types, respectively. The patients with abnormal brain scan having PCS demonstrated a significant difference in the frequency of both brain lesions site $(P<0.001)$ and type $(P<0.025)$ versus those without PCS. It was revealed that accompanied hematoma (52.25\%) and fronto-temporal lesion (34.23\%) in PCS patients were more than those in non-PCS ones. The frontal lesion was the most prevalent lesion site in both PCS and non-PCS groups, however, the frequency of its manifestations on brain scans was relatively similar in both groups. There was a significant difference in lesion plurality between PCS and non-PCS groups $(P<0.032)$. Multifocal brain lesions mainly emerged on the brain scan of $\mathrm{mTBI}$ patients with PCS $(47.74 \%)$ compared with those without PCS (27.53\%) (Table 2).

The age range of both $\mathrm{mTBI}$ patients with and without PCS was 20-35 years (Figure 2). According to the results of the multivariate logistic regression analysis, the existence of pathology on the early brain scan was the first strong predictor of PCS. Abnormal findings on the initial brain scans dramatically increased the risk of PCS occurrence by 7.2 times $(P<0.001)$. Fronto-temporal lesion and accompanied hematoma were second and third potential factors which respectively anticipated the development of PCS. Substantially, the fronto-temporal lesion enhanced the risk of PCS by 4.1 times $(P<0.017)$. The risk of PCS development was elevated by 2.6 times in those with a manifestation of multiple hematomas on their initial brain scan (Table 3).

\section{Discussion}

The results of the present study signified that initial brain scan data on admission was a potential strong predictor of PCS development after mTBI. Likewise, the demographic factors, clinical and TBI-related variables such as non-brain traumatic injuries, duration of hospi- talization, past medical diseases, the functional outcome at discharge, GCS score, and mechanism of head trauma were not associated with PCS appearance. It seems that a negligible association of demographic and clinical variables with PCS might be due to insufficient sample size in this study.

According to a previous study, the age range between 25-30 years old had more frequency in mTBI patients with PCS [21]. We quantified the common age range in both PCS and non-PCS groups between 20 to 35 years. Therefore, no meaningful association was found between PCS and the age range that might be due to more prevalence of this age range in the total mTBI population. As a possible explanation, the discrepancy in the statistical significance of the association between age and PCS in the current study and prior study can be attributed to the diversity in the communities under study.

Another prior investigation in Iran reported similar findings according to which most of mTBI patients' age ranged from 21 to 30 years (22]. It seems that this age range is the active population in our society who might considerably be at the risk of $\mathrm{mTBI}$. Our data regarding the poor relationship between gender and trauma mechanism with PCS was compatible with the results of previous studies $[23,24]$.

In the past, a study revealed that $\mathrm{MTBI}$ patients with a pathological lesion on initial brain CT scan having PCS demonstrated significant differences in terms of GCS score. In other words, the frequency of GCS scores of 13 and 14 in mTBI patients with PCS was higher in the prior study [25]. However, we did not find any significant relationship between GCS score and PCS occurrence. A possibility to explain this discrepancy might be attributed to the difference in comparable groups of previous studies and our project. In the prior study, the comparison of GCS score was done between PCS patients with and without brain abnormality, while we compared the GCS score between mTBI patients with and without PCS. In our study, all subjects with PCS except for 2 patients had an abnormal brain scan. Therefore, this type of classification was useless in our study. 


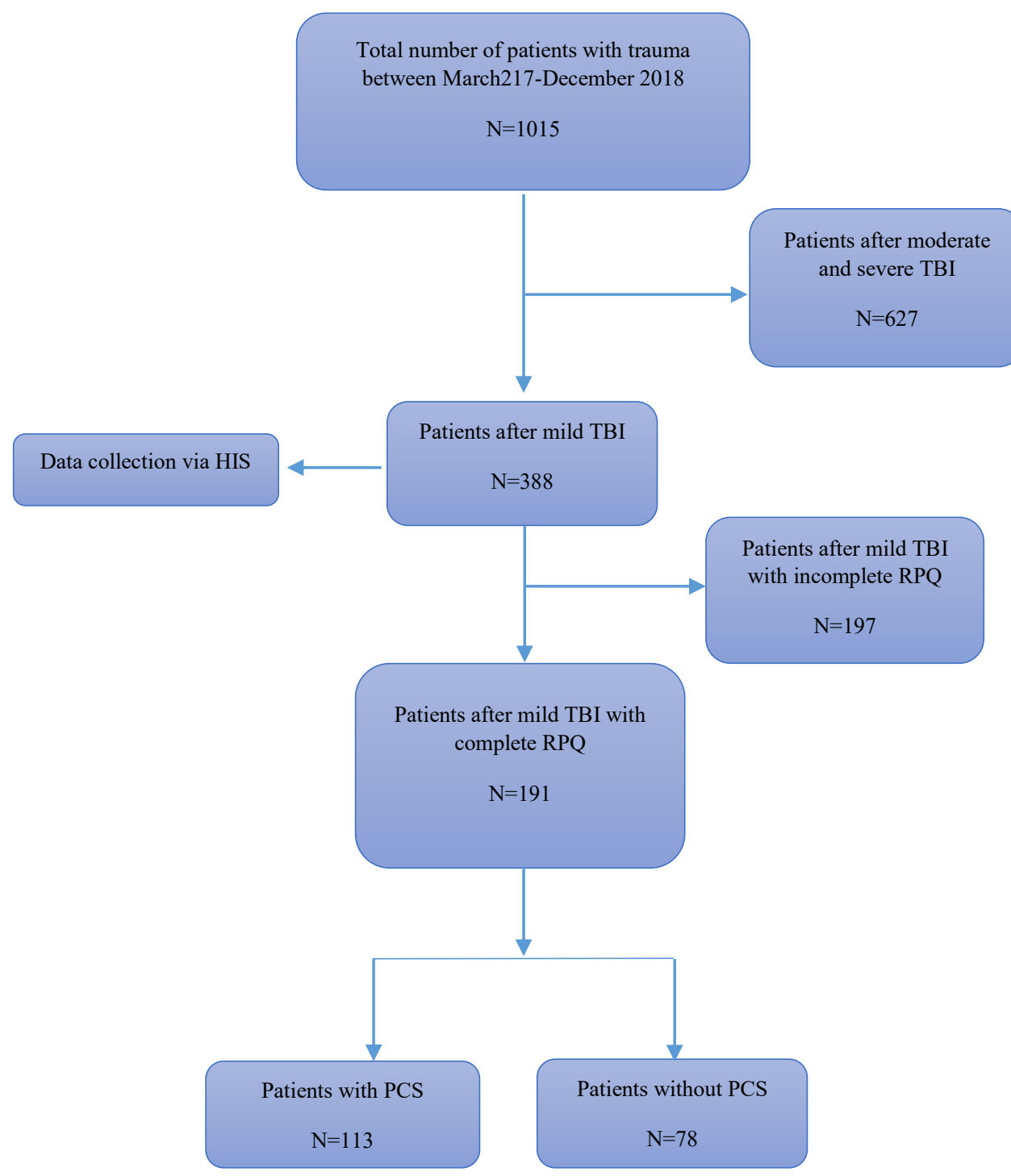

Figure 1. Algorithm of study from mTBI identification to PCS diagnosis

In the current study, there was a significant relationship between PCS and past mental and mood disorders which is compatible with a previous study [26]. It seems that previous mental and psychological disorders may predispose $\mathrm{mTBI}$ patients to manifest the PCS signs. Besides, it might be hypothesized that the prior mental and psychological disorders disturb some neural circuits which might be more susceptible to prolonged detrimental consequences induced by TBI [27]. Thus, worsening impaired neural circuits due to delayed secondary insults of $\mathrm{mTBI}$ over time may increase the development of psychological symptoms of PCS. Further studies are required to prove this conclusion. Accordingly, recording the specification of past psychological diseases in detail and follow-up neuroimaging are strongly recommended to detect these issues in advance.
Another finding of our study was that accompanied non-brain traumatic injury in $\mathrm{mTBI}$ patients was not the risk factor of PCS. Some researchers compared PCS appearance between only $\mathrm{mTBI}$ patients and only orthotropic ones in the past [28]. The results of their investigation clarified that PCS significantly was different between these 2 groups. The different scales and methods for the determination of PCS between the previous study and our work seem to be the reason for this discrepancy. We realized that functional outcomes at discharge had no potential to predict PCS appearance in $\mathrm{mTBI}$ patients. The evidence of a prior study has corroborated our results in this regard [29].

An important finding of this study was that abnormal brain scans especially multifocal hematoma in frontal 

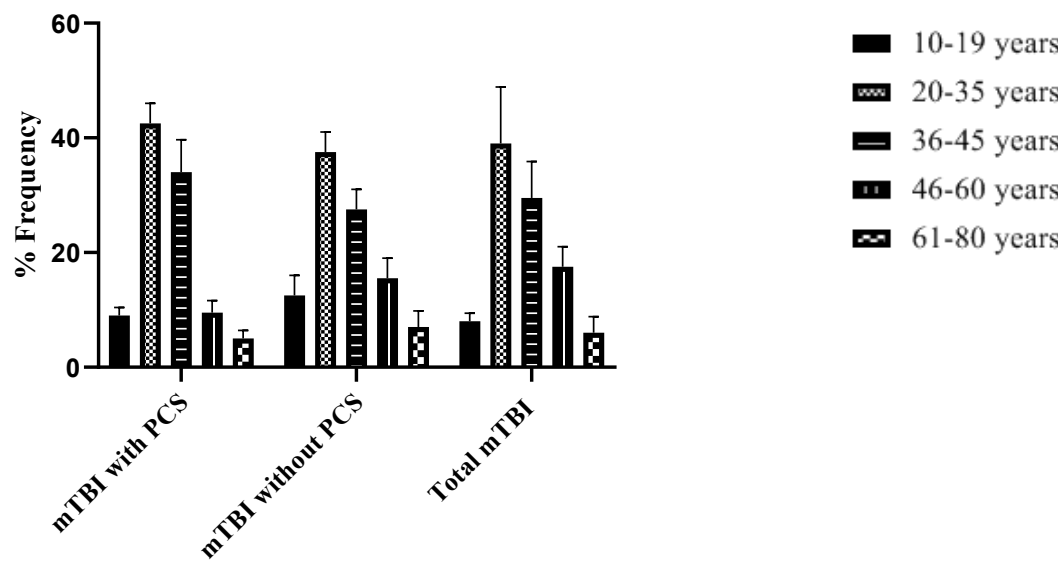

Figure 2. The prevalence of age range in indicated groups

and temporal cerebral lobes increased the risk of developing PCS after mTBI. In accordance with our results, a significant association between PCS and pathological lesions on brain scans of $\mathrm{MTBI}$ patients has been reported by some authors $[23,25,30]$. It seems that some biological subsequences, including inflammation, ischemia, excitotoxicity, apoptosis, and gliosis, in the central nervous system may occur after mTBI leading to neural impairment, neural network inefficiency, and a disturbance of the neurotransmitter balance and participated in the physical and mental problems reported by the patients with PCS [31]. The subtle neural abnormalities in minor TBIs can mainly be found by advanced MRI techniques [32-35]. These neurobiological events called secondary insults may negatively influence the cognitive, affective, and emotional and sleep processing in the brain and continue for days or even months after primary mechanical injury onset $[31,36,37]$. The secondary brain damages gradually occur and become widespread, thus it might be suggested that early therapeutic approaches may protect neural structure and function by keeping from the progression of harmful neurobiological processes in the lesions and their surrounding tissue. Fundamentally, frontal and temporal lobes are the vulnerable anatomic regions after $\mathrm{TBI}[38,39]$.

The damages of these cerebral lobes can impact the principle neural functions involved in executive function, cognitive and emotional perception, learning, and high-level mental processing $[36,38]$ and bring about cognitive and affective manifestations of PCS. Notably, multifocal lesions having at least one of the hematoma types appear to expand the deleterious neurobiological processes and deteriorate brain function for a long time, causing significant clinical post-concussion symptoms. Although subjective examination of PCS is applicable through recording the patient's statements us- ing various types of questionnaires and checklists, PCS subjective labeling may usually result from patient's simulation for the reasons of litigation and legal claims. Thus, a definitive diagnosis of the PCS due to TBI can be obtained by combining the initial brain scan data and patient's responses to questionnaires evaluating PCS.

\section{Conclusion}

The current study represented the significance of initial abnormal brain scan especially multifocal hematoma in the frontal and temporal cerebral lobes in the prediction of PCS post MTBI. Here, we found that previous psychological disease was associated with PCS. However, no association was observed between PCS occurrence with demographic data, TBI-related clinical features, and past medical history. Therefore, followup care for $\mathrm{mTBI}$ patients at the risk of PCS is strictly recommended to prevent persistent post-concussion symptoms. Furthermore, brain scan data appears to be useful as an objective measure for the detection of mTBI-induced PCS.

\section{Ethical Considerations}

\section{Compliance with ethical guidelines}

All ethical principles are considered in this article. The participants were informed of the purpose of the research and its implementation stages. They were also assured about the confidentiality of their information and were free to leave the study whenever they wished, and if desired, the research results would be available to them.

\section{Funding}

This project was funded by Guilan Road Trauma Research Center (Grant No.: 971213/68). 


\section{Authors' contributions}

Conception and design: Sara Ramezani; Data collection: Seddigheh Eslamparast, Zoheir Reihanian; Data analysis and interpretation: Sara Ramezani; Drafting the article: Seddigheh Eslamparast; Critically revising the article: Sara Ramezani; Reviewing submitted version of manuscript: Sara Ramezani; Approving the final version of the manuscript: Sara Ramezani.

\section{Conflict of interest}

The authors declared no conflict of interest.

\section{Acknowledgments}

We thank Guilan Road Trauma Research Center and Clinical Research Development Unit of Poursina Hospital, Guilan University of Medical Sciences for supporting us in data collection.

\section{References}

[1] Langlois JA, Rutland-Brown W, Wald MM. The epidemiology and impact of traumatic brain injury: A brief overview. The Journal of Head Trauma Rehabilitation. 2006; 21(5):375-8. [DOI:10.1097/00001199-200609000-00001] [PMID]

[2] Ragnarsson KT. Results of the NIH consensus conference on" rehabilitation of persons with traumatic brain injury". Restorative Neurology and Neuroscience. 2002; 20(3-4):103-8. https:/ / content.iospress.com/articles/restorative-neurology-and-neuroscience/rnn00199

[3] Council NR. Gulf War and health: volume 7: Long-term consequences of traumatic brain injury. Washington, DC: National Academies Pr; 2008.

[4] Meerhoff S, De Kruijk J, Rutten J, Leffers P, Twijnstra A. Incidence of traumatic head or brain injuries in catchment area of Academic Hospital Maastricht in 1997. Nederlands Tijdschrift voor Geneeskunde. 2000; 144(40):1915-8. [PMID]

[5] Thornhill S, Teasdale GM, Murray GD, McEwen J, Roy CW, Penny KI. Disability in young people and adults one year after head injury: Prospective cohort study. The BMJ. 2000; 320(7250):1631-5. [DOI:10.1136/bmj.320.7250.1631] [PMID] [PMCID]

[6] Ingebrigtsen T, Romner B. Cognitive function in patients with increased serum levels of protein S-100 after minor head injury. Archives of Clinical Neuropsychology. 1997; 4(12):424. [DOI:10.1016/S0887-6177(97)83723-7]

[7] Herrmann M, Curio N, Jost S, Grubich C, Ebert A, Fork M, et al. Release of biochemical markers of damage to neuronal and glial brain tissue is associated with short and long term neuropsychological outcome after traumatic brain injury. Journal of Neurology, Neurosurgery \& Psychiatry. 2001; 70(1):95-100. [DOI:10.1136/jnnp.70.1.95] [PMID] [PMCID]
[8] Papa L, Edwards D, Ramia M. Exploring Serum Biomarkers for Mild Traumatic Brain Injury. In: Kobeissy FH, editor. Brain Neurotrauma: Molecular, Neuropsychological, and Rehabilitation Aspects. Boca Raton (FL): CRC Press/Taylor \& Francis; 2015 [PMID]

[9] Binder LM. Persisting symptoms after mild head injury: A review of the postconcussive syndrome. Journal of Clinical and Experimental Neuropsychology. 1986; 8(4):323-46. [DOI:10.1080/01688638608401325] [PMID]

[10] Cooper J. Diagnostic and statistical manual of mental disorders ( $4^{\text {th }}$ edn, text revision)(DSM-IV-TR) Washington, DC: American Psychiatric Association 2000. [DOI:10.1192/bjp.179.1.85-a]

[11] Leddy JJ, Sandhu H, Sodhi V, Baker JG, Willer B. Rehabilitation of concussion and post-concussion syndrome. Sports Health 2012; 4(2):147-54. [DOI:10.1177/1941738111433673] [PMID] [PMCID]

[12] Levin HS, Diaz-Arrastia RR. Diagnosis, prognosis, and clinical management of mild traumatic brain injury. The Lancet Neurology. 2015; 14(5):506-17. [DOI:10.1016/S1474-4422(15)00002-2]

[13] Murray L, Teasdale G, Jennett B, Kelly D, Murray G, Miller $\mathrm{J}$, et al. Does prediction of outcome alter patient management? The Lancet. 1993; 341(8859):1487-91. [DOI:10.1016/01406736(93)90631-P]

[14] Perel P, Wasserberg J, Ravi RR, Shakur H, Edwards P, Roberts I. Prognosis following head injury: A survey of doctors from developing and developed countries. Journal of Evaluation in Clinical Practice. 2007; 13(3):464-5. [DOI:10.1111/j.13652753.2006.00713.x] [PMID]

[15] World Health Organization (WHO). The ICD-10 classification of mental and behavioural disorders: Diagnostic criteria for research. Geneva: World Health Organization; 1993. https:// www.who.int/classifications/icd/en/GRNBOOK.pdf

[16] King N, Crawford S, Wenden F, Moss N, Wade D. The Rivermead Post Concussion Symptoms Questionnaire: A measure of symptoms commonly experienced after head injury and its reliability. Journal of Neurology. 1995; 242(9):587-92. [DOI:10.1007/ BF00868811] [PMID]

[17] Ruff RM. Mild traumatic brain injury and neural recovery: Rethinking the debate. Neuro Rehabilitation. 2011; 28(3):167-80 [DOI:10.3233/NRE-2011-0646] [PMID]

[18] Khong E, Odenwald N, Hashim E, Cusimano MD. Diffusion tensor imaging findings in post-concussion syndrome patients after mild traumatic brain injury: A systematic review. Frontiers in Neurology. 2016; 7:156. [DOI:10.3389/fneur.2016.00156] [PMID] [PMCID]

[19] Hiploylee C, Dufort PA, Davis HS, Wennberg RA, Tartaglia MC, Mikulis D, et al. Longitudinal study of postconcussion syndrome: not everyone recovers. Journal of Neurotrauma. 2017 34(8):1511-23. [DOI:10.1089/neu.2016.4677] [PMID] [PMCID]

[20] Potter S, Leigh E, Wade D, Fleminger S. The rivermead post concussion symptoms questionnaire. Journal of Neurology. 2006; 253(12):1603-14. [DOI:10.1007/s00415-006-0275-z] [PMID]

[21] Tator $\mathrm{CH}$, Davis HS, Dufort PA, Tartaglia MC, Davis KD, Ebraheem A, et al. Postconcussion syndrome: Demographics and predictors in 221 patients. Journal of Neurosurgery. 2016; 125(5):1206-16. [DOI:10.3171/2015.6.JNS15664] [PMID] 
[22] Vafaee R, Vafaei A, Forouzanfar MM, Asadollahi S, Kashani $P$, Heidari K, et al. Epidemiology of traumatic brain injury in Iranian population: The results of a multicenter study. Wulfenia. 2013; 20(9):257-63. https:/ / www.researchgate.net/ profile/Reza_Vafaee2/publication/257240081_

[23] Heidari K, Asadollahi S, Jamshidian M, Abrishamchi SN, Nouroozi M. Prediction of neuropsychological outcome after mild traumatic brain injury using clinical parameters, serum S100B protein, and findings on computed tomography. Brain Injury. 2015; 29(1):33-40. [DOI:10.3109/02699052.2014.948068 ] [PMID]

[24] Mickevičiene D, Schrader H, Obelieniene D, Surkiene D, Kunickas R, Stovner LJ, et al. A controlled prospective inception cohort study on the post-concussion syndrome outside the medicolegal context. European Journal of Neurology. 2004; 11(6):411-9. [DOI:10.1111/j.1468-1331.2004.00816.x] [PMID]

[25] Voormolen DC, Haagsma JA, Polinder S, Maas AI, Steyerberg EW, Vuleković P, et al. Post-concussion symptoms in complicated vs. uncomplicated mild traumatic brain injury patients at three and six months post-injury: Results from the center-tbi study. Journal of Clinical Medicine. 2019; 8(11):1921. [DOI:10.3390/jcm8111921] [PMID] [PMCID]

[26] Morgan CD, Zuckerman SL, Lee YM, King L, Beaird S, Sills AK, et al. Predictors of postconcussion syndrome after sportsrelated concussion in young athletes: A matched case-control study. Journal of Neurosurgery: Pediatrics. 2015; 15(6):589-98. [DOI:10.3171/2014.10.PEDS14356] [PMID]

[27] Yousefzadeh-Chabok S, Ramezani S, Reihanian Z, Safaei M, Alijani B, Amini N. The role of early posttraumatic neuropsychological outcomes in the appearance of latter psychiatric disorders in adults with brain trauma. Asian Journal of Neurosurgery. 2015; 10(3):173. [DOI:10.4103/17935482.161165] [PMID] [PMCID]

[28] McCauley SR, Wilde EA, Barnes A, Hanten G, Hunter JV, Levin HS, et al. Patterns of early emotional and neuropsychological sequelae after mild traumatic brain injury. Journal of Neurotrauma. 2014; 31(10):914-25. [DOI:10.1089/ neu.2012.2826] [PMID] [PMCID]

[29] Bown D, Belli A, Qureshi K, Davies D, Toman E, Upthegrove R. Post-traumatic stress disorder and self-reported outcomes after traumatic brain injury in victims of assault. PloS One. 2019; 14(2):e0211684. [DOI:10.1371/journal. pone.0211684] [PMID] [PMCID]

[30] Lange RT, Iverson GL, Brubacher JR, Mädler B, Heran MK. Diffusion tensor imaging findings are not strongly associated with postconcussional disorder 2 months following mild traumatic brain injury. Journal of Head Trauma Rehabilitation. 2012; 27(3):188-98. [DOI:10.1097/HTR.0b013e318217f0ad] [PMID]

[31] McAllister TW. Neurobiological consequences of traumatic brain injury. Dialogues in Clinical Neuroscience. 2011 13(3):287-300. [DOI:10.31887/DCNS.2011.13.2/tmcallister] [PMID] [PMCID]

[32] Hellstrøm T, Westlye LT, Kaufmann T, Doan NT, Søberg $\mathrm{HL}$, Sigurdardottir S, et al. White matter microstructure is associated with functional, cognitive and emotional symptoms 12 months after mild traumatic brain injury. Scientific Reports. 2017; 7(1):1-14. [DOI:10.1038/s41598-017-13628-1] [PMID] [PMCID]
[33] King R, Grohs MN, Kirton A, Lebel C, Esser MJ, Barlow KM. Microstructural neuroimaging of white matter tracts in persistent post-concussion syndrome: A prospective controlled cohort study. NeuroImage: Clinical. 2019; 23:101842. [DOI:10.1016/j.nicl.2019.101842] [PMID] [PMCID]

[34] Narayana PA. White matter changes in patients with mild traumatic brain injury: MRI perspective. Concussion. 2017; 2(2):CNC35. [DOI:10.2217/cnc-2016-0028] [PMID] [PMCID]

[35] Puig J, Ellis MJ, Kornelsen J, Figley TD, Figley CR, Daunisi-Estadella $\mathrm{P}$, et al. Magnetic resonance imaging biomarkers of brain connectivity in predicting outcome after mild traumatic brain injury: A systematic review. Journal of Neurotrauma. 2020; 37(16):1761-6. [DOI:10.1089/neu.2019.6623] [PMID]

[36] Ramezani S, Reihanian Z, Hosseini Nejad M, YousefzadehChabok S. Neuropsychological and neuropsychiatric deficits following traumatic brain injury: Common patterns and neuropathological mechanisms. Iranian Journal of Neurosurgery. 2018; 4(4):185-98. http:/ /irjns.org/article-1-161-en.html

[37] Ramezani S, Yousefzadeh-Chabok S. Identification of imaging and clinical markers predicting chronic sleep disturbances after traumatic brain injury in adults. Iranian Journal of Neurosurgery. 2018; 4(3):125-38. [DOI:10.32598/irjns.4.3.125]

[38] Chabok SY, Kapourchali SR, Leili EK, Saberi A, Mohtasham-Amiri Z. Effective factors on linguistic disorder during acute phase following traumatic brain injury in adults. Neuropsychologia. 2012; 50(7):1444-50. [DOI:10.1016/j.neuropsychologia.2012.02.029] [PMID]

[39] Chabok SY, Kapourchali SR, Saberi A, Mohtasham-Amiri $\mathrm{Z}$. Operative and nonoperative linguistic outcomes in brain injury patients. Journal of the Neurological Sciences. 2012; 317(1-2):130-6. [DOI:10.1016/j.jns.2012.02.009] [PMID] 